\title{
Fratura do escafoide társico em um praticante de parkour, uma lesão rara - Relato de Caso e Revisão da Literatura*
}

\section{Tarsal Navicular Fracture in a Parkour Practitioner, a Rare Injury - Case Report and Literature Review}

\author{
Samir Karmali ${ }^{1}$ Jorge Teixeira Ramos ${ }^{1}$ João Almeida ${ }^{1}$ André Barros ${ }^{1}$ Pedro Campos ${ }^{1}$ \\ Daniel Sá da Costa ${ }^{1}$ \\ ${ }^{1}$ Departamento de Cirurgia Ortopédica, Hospital Vila Franca de Xira, \\ Vila Franca de Xira, Portugal \\ Rev Bras Ortop 2019;54:739-745. \\ Endereço para correspondência: Samir Karmali, Departamento de \\ Cirurgia Ortopédica, Hospital Vila Franca de Xira, Rua Calouste \\ Gulbenkian 1, 2600-009, Vila Franca de Xira, Portugal \\ (e-mail: samirkarmali@hotmail.com).
}

\section{Resumo \\ Palavras-chave \\ - fraturas ósseas \\ - ossos do tarso \\ - articulações társicas \\ - esportes \\ - procedimentos/ métodos ortopédicos}

\author{
Abstract \\ Keywords \\ - bone fractures \\ - tarsal bones \\ - tarsal joints \\ - sports \\ - orthopedic \\ procedures/methods
}

As fraturas do escafoide társico, bem como outras lesões do médiopé, são raras e podem resultar em incapacidade grave se não forem tratadas adequadamente. Parkour, um esporte moderno, está ganhando popularidade entre os jovens em áreas urbanas, e é propenso a traumatismos de alta energia, sendo estes escassamente descritos na literatura. O presente relato trata de um caso de fratura rara do escafoide társico em um paciente do sexo masculino de 17 anos, ocorrida durante a prática de parkour, que foi tratada com sucesso, com redução aberta e fixação interna. A descrição do caso enfatiza os desafios na sua abordagem; a discussão destaca as opções de tratamento e seus objetivos. O caso também deve alertar sobre a ocorrência crescente dessas lesões incomuns.

Tarsal navicular fractures, as well as other midfoot injuries, are rare, and can result in severe impairment if not properly treated. Parkour, a modern sport, is gaining popularity among young individuals in urban areas, and is prone to result in highenergy trauma, which is scarcely described in the literature. The following is a report of a rare case of tarsal navicular fracture in a 17-year-old male, sustained during parkour practice, which was treated successfully with open reduction and internal fixation. The description of the case emphasizes the challenges of its approach; the discussion highlights the treatment options and goals. The case should also raise awareness about the increasing occurrence of these uncommon lesions.

\section{Introdução}

O escafoide társico, um osso em forma de crescente no médiopé, é também chamado de "navicular" devido à sua semelhança com um barco [em latim, "navis"]. ${ }^{1}$ Tem papel

Trabalho realizado no Departamento de Cirurgia Ortopédica, Hospital Vila Franca de Xira, Vila Franca de Xira, Portugal. Originalmente Publicado por Elsevier Editora Ltda.

recebido

08 de Novembro de 2017 aceito

23 de Dezembro de 2017
DOI https://doi.org/

10.1016/j.rboe.2017.12.009. ISSN 0102-3616. fundamental na coluna medial do pé, suportando a maior parte da carga axial no médiopé durante o apoio de peso e o impulso; ${ }^{2,3}$ também faz parte das articulações de Lisfranc (naviculocuneiforme) e Chopart (talonavicular), esta última responsável por quase $80 \%$ do movimento do retropé..$^{1-8}$ Devido às suas características anatômicas e funcionais específicas, bem como seu suprimento sanguíneo radial tênue, $3,5,9,10$ as lesões do osso escafoide (ou no médiopé) podem ter consequências graves e incapacitantes. Felizmente, as lesões
Copyright $\odot 2019$ by Sociedade Brasileira License terms de Ortopedia e Traumatologia. Published by Thieme Revinter Publicações Ltda, Rio de Janeiro, Brazil 
no médiopé são raras (cerca de 5\% de todas as lesões no pé), ${ }^{6}$ e a incidência geral de fraturas nessa área é de $0,45 \% .{ }^{11}$ O subgrupo de fraturas do escafoide é incomum, e essa lesão é ainda mais rara. $^{2,3}$ Embora a maioria dessas lesões seja de fratura por avulsão (que representam quase $50 \%$ das fraturas do escafoide), ${ }^{3,5,12}$ a literatura descreve uma frequência maior de fraturas mais complexas e de alta energia (decorrentes de acidentes com veículos automotores, lesões esportivas e quedas). ${ }^{1,2,4,6}$

Recentemente, o parkour surgiu como um esporte radical em que os praticantes (traceurs) pretendem ir de um ponto a outro em um cenário complexo (geralmente áreas urbanas) da maneira mais rápida e eficiente possível, sem equipamento auxiliar. ${ }^{13-15}$ Apesar de ser uma modalidade propensa a lesões complexas e de alta energia, há poucos casos descritos na literatura.

Os autores relatam um caso raro de fratura do escafoide társico durante a prática de parkour.

\section{Relato de Caso}

Um rapaz de 17 anos de idade, estudante do Ensino Médio e traceur, sem histórico médico relevante, foi atendido em nosso departamento de emergência pediátrica (em março de 2016) depois de sofrer uma queda de 3,5 m ao pular de um prédio (primeiro andar) durante um exercício de parkour. $\mathrm{O}$ paciente se queixou apenas de dor no pé direito, que foi a superfície de contato de aterrissagem na queda, quando estava em posição de flexão plantar e abdução do antepé. Clinicamente, o pé apresentava edema proeminente de partes moles, e a avaliação neurovascular não mostrou comprometimento; a ocorrência de síndrome compartimental foi excluída. Um conjunto completo de radiografias do pé e do tornozelo (projeções anteroposterior, lateral e oblíqua medial a $30^{\circ}$ de Myerson) foi realizado, e mostrou uma fratura do escafoide társico com deslocamento e cominuição articular, perda de altura do arco medial e encurtamento da coluna medial do pé (classificada como Sangeorzan tipo III/OTA 74 tipo C), sem outras lesões aparentes, o que foi confirmado por tomografia computadorizada (TC) multiaxial e de reconstrução tridimensional (3D) (-Fig. 1). Devido às más condições dos tecidos moles, a fixação externa com ligamentotaxia tíbio-calcâneo-cuboide-metatársica foi realizada (-Fig. 2). Duas semanas depois (sob anestesia geral, com uso de torniquete e posicionamento supino), por meio de uma abordagem dorsal (imediatamente lateral à artéria

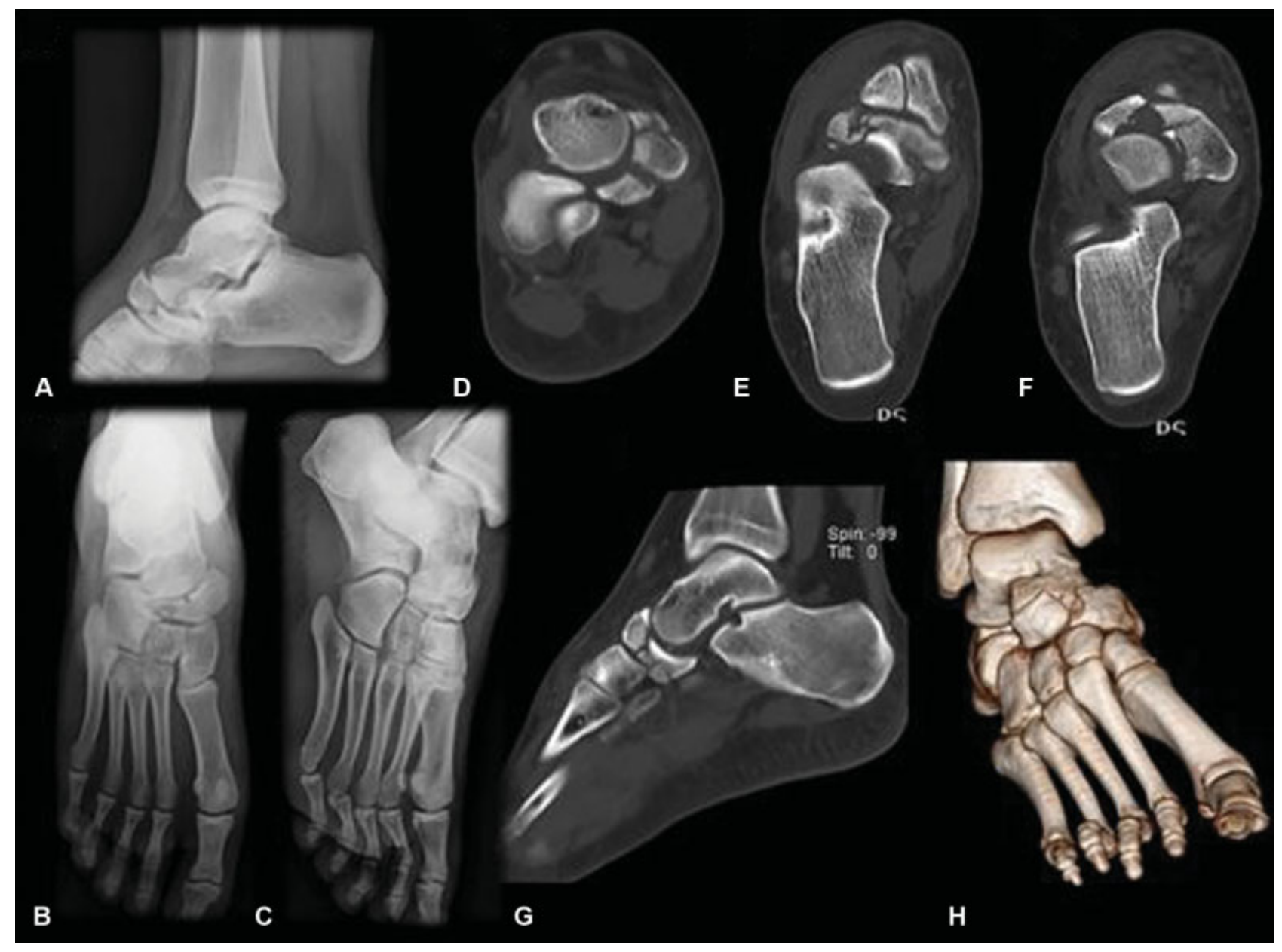

Fig. 1 Estudos de imagem do pé e do tornozelo direitos mostrando uma fratura do escafoide társico com deslocamento e cominuição articular, perda de altura do arco medial, e encurtamento da coluna medial do pé (classificada como Sangeorzan tipo III/OTA 74 tipo C). (A) Radiografia lateral do tornozelo direito; (B) projeção anteroposterior do pé direito; (C) projeção oblíqua medial em 30。 do pé direito; (D) tomografia computadorizada (TC) axial; (E) e (F) TC coronal; (G) TC sagital; (H) TC com reconstrução tridimensional. 


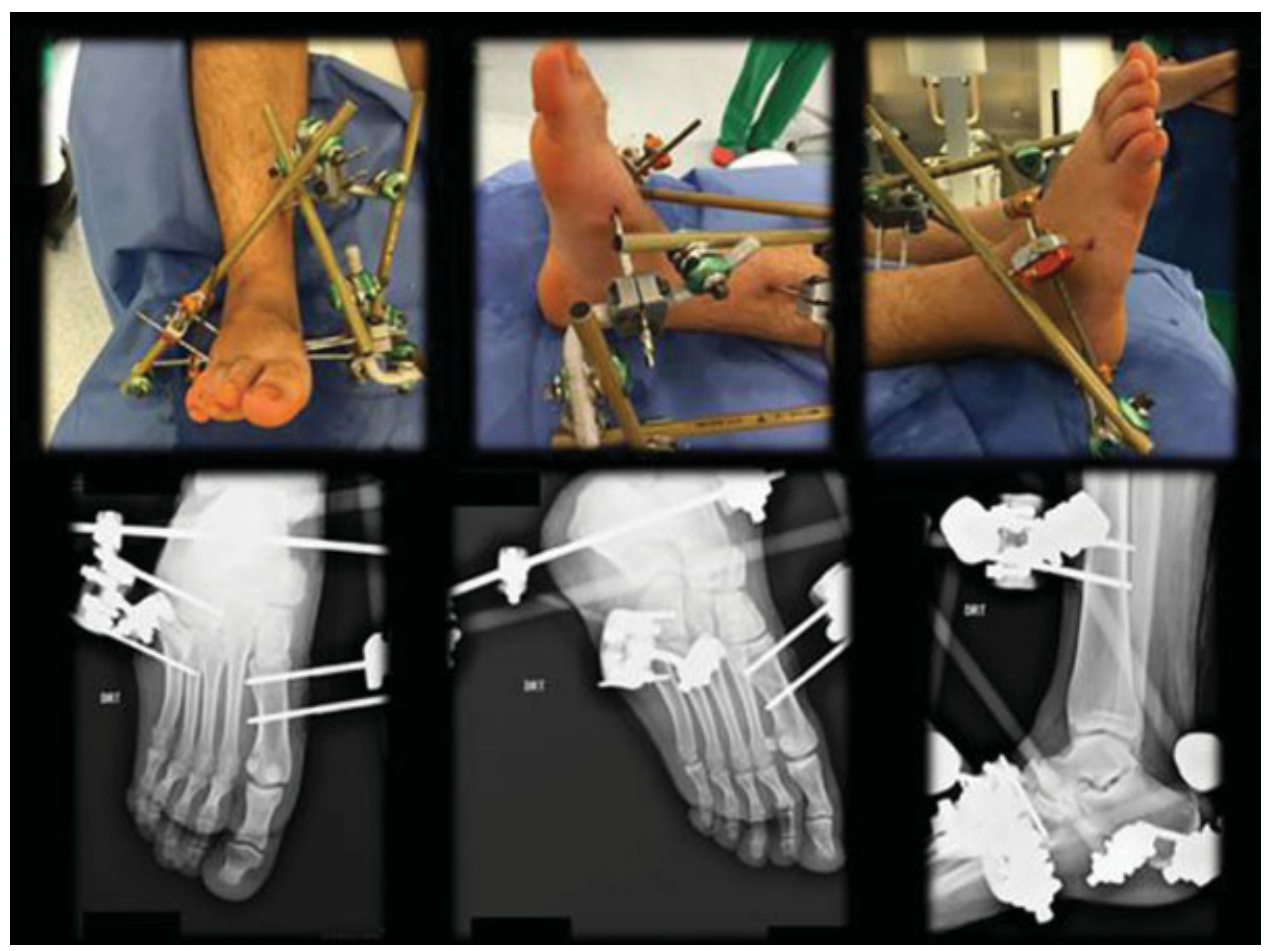

Fig. 2 Ligamentotaxia tíbio-calcâneo-cuboide-metatársica (superior: aparência externa; inferior: avaliação radiológica).

pedicular dorsal), com incisão medial minimamente invasiva (entre os tendões anterior e posterior tibial), o paciente foi submetido a redução aberta e fixação interna (RAFI) com placa anatômica e fixação de extensão externa adjunta (com colocação de pino no colo do tálus e no primeiro metatarso) (-Fig. 3). O paciente foi encaminhado para atendimento ambulatorial com indicação para não sustentação de peso por seis semanas. Após esse período, a fixação externa foi removida, e a sustentação parcial do peso foi permitida com incrementos progressivos de acordo com as avaliações clínicas e radiológicas. Quinze semanas após a RAFI, a avaliação radiológica mostrou sinais de cicatrização da fratura (-Fig. 4), e atividade física sem restrições foi permitida.

Às 34 semanas de acompanhamento, a pontuação no American Orthopedic Foot and Ankle Score (AOFAS) foi de 87 (de 100 pontos). Clinicamente, o paciente apresentava dor mínima e ocasional. Não foram observadas alterações de união ou artrite pós-traumática. Durante o tratamento, a

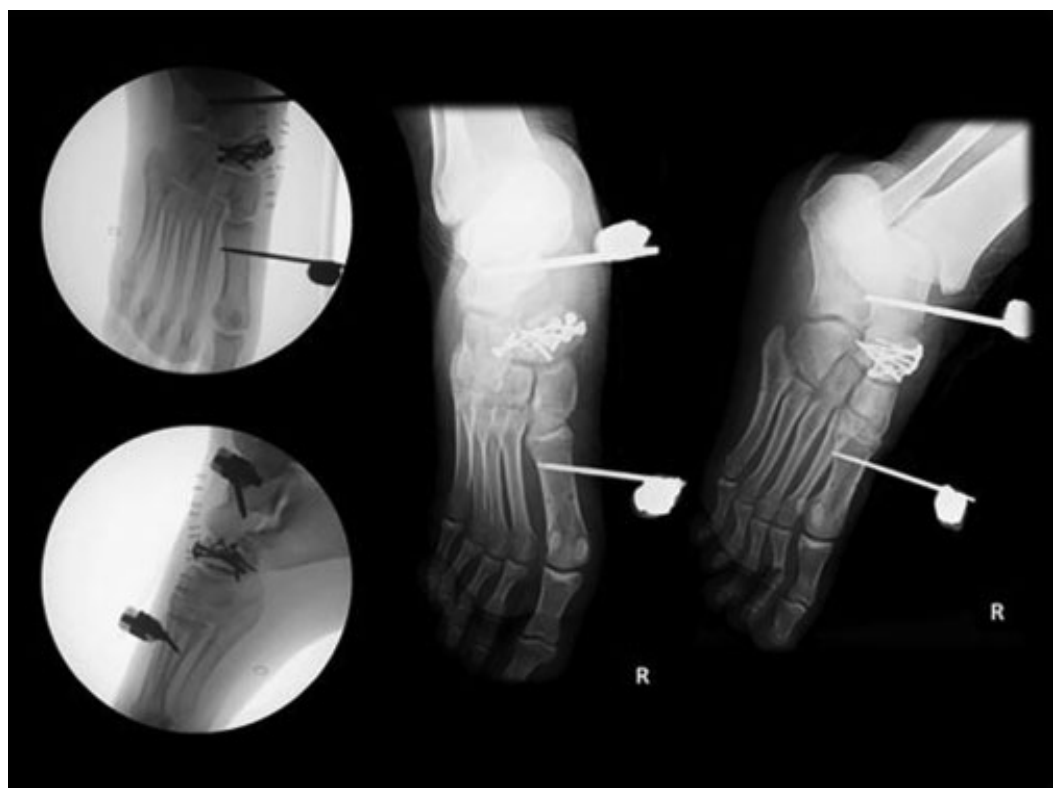

Fig. 3 Redução aberta e fixação interna com placa anatômica pré-contornada e fixação de extensão externa auxiliar (com colocação de pino no colo do tálus e no primeiro metatarso). 


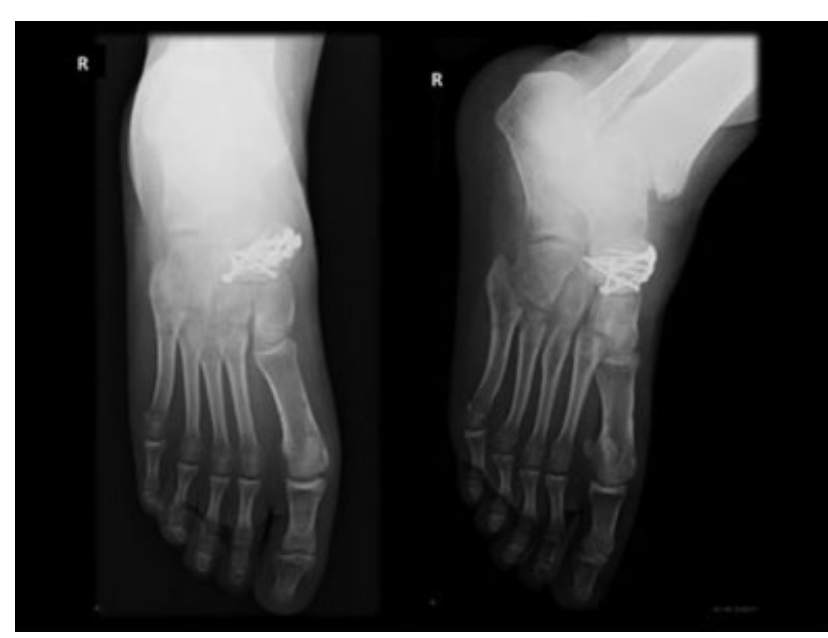

Fig. 4 Cicatrização da fratura (às 15 semanas após a cirurgia).

integridade dos tecidos moles foi uma questão importante, principalmente logo após a RAFI. No entanto, por fim, não foram observadas complicações, e o resultado estético foi bom. Do ponto de vista biomecânico, não houve comprometimento de atividades de alta demanda, como corrida, apesar de uma ligeira limitação de inversão em comparação ao pé contralateral. Ao exame clínico, o paciente apresentava marcha normal, e não havia alinhamento em varo/valgo ou deformidade em pé cavo/plano em comparação ao pé contralateral (-Fig. 5).

O paciente ficou muito satisfeito com o resultado, e, um ano após a RAFI, realizava atividades como escalada em rocha sem queixas ou limitações (AOFAS $=90$ pontos).

\section{Discussão}

Há poucas lesões associadas ao parkour descritas na literatura; ${ }^{13-15}$ porém, um estudo transversal retrospectivo ${ }^{13}$ mostrou que a maioria das lesões afeta o membro superior (58\%), em especial sua região distal; no membro inferior (27\%), a distribuição das lesões ocorre de maneira oposta. A aterrisagem é o movimento responsável pela maioria das lesões (61\%). ${ }^{13}$

Como já mencionado, as fraturas do escafoide társico são raras. Em 1989, Sangeorzan et $\mathrm{al}^{4}$ classificaram as fraturas do escafoide társico de acordo com a direção da linha de fratura, o padrão de ruptura das articulações adjacentes, e a direção do deslocamento do pé; assim, quatro tipos principais fraturas de tuberosidade, por avulsão capsular, por estresse, e do corpo navicular (essas últimas são divididas em três subtipos) - foram estabelecidos, bem como uma relação entre a gravidade da lesão e o resultado funcional. Comparadas às fraturas por avulsão e estresse (que são as menos raras), as fraturas articulares cominutivas com deslocamento do corpo navicular (tipo III) são geralmente provocadas por traumas de alta energia, e prejudicam a estabilidade da coluna medial do pé. ${ }^{16-19}$ Estas últimas (assim como as fraturas de tipo II) são frequentemente associadas a outras lesões do médiopé (por exemplo, da articulação calcaneocuboidea e dos ligamentos naviculocuneiformes, que devem ser excluídas); nesses casos, a sustentação da carga axial pelo escafoide é geralmente feita com o antepé em flexão e adução plantares (nas fraturas de tipo II) ou abdução (nas fraturas de tipo III), ${ }^{1-4,12}$ como ocorreu neste caso.

Clinicamente, as fraturas do escafoide társico podem ser observadas tanto em pés quase normais quanto em membros inferiores com lesões graves. $\mathrm{O}$ alto índice de suspeita e o

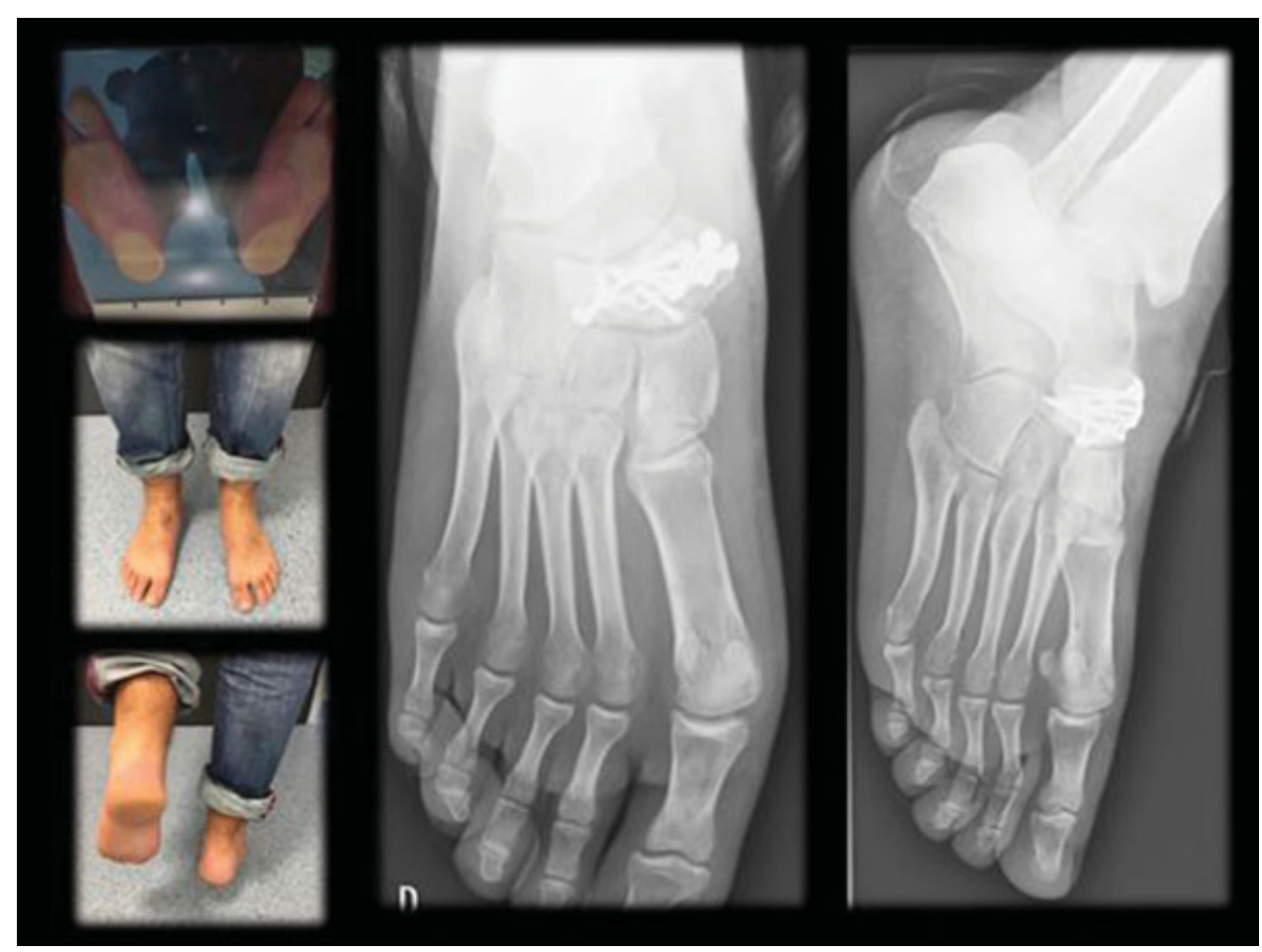

Fig. 5 Às 34 semanas de acompanhamento: exame clínico (sem deformidade em cavo/plano ou varo/valgo) e avaliação radiológica. 
conhecimento das possíveis lesões graves (como da síndrome compartimental) devem ser abordados imediatamente. A avaliação radiológica é crucial no estabelecimento do diagnóstico. Embora os exames radiográficos comuns (inclusive projeção oblíqua medial - por exemplo, uma projeção oblíqua de $30^{\circ}$ de Myerson) sejam suficientes para fazer o diagnóstico, ${ }^{20}$ o uso da tomografia computadorizada (TC) é inestimável para melhor caracterização da fratura e das luxações perinaviculares (para o planejamento do tratamento cirúrgico), bem como para exclusão de lesões associadas e variantes anatômicas do escafoide; ${ }^{21}$ na verdade, um estudo ${ }^{22}$ demonstrou que a sensibilidade da radiografia primária (no diagnóstico de uma fratura do escafoide társico) foi 33\% maior do que a da TC.

O tratamento dessas fraturas visa assegurar a integridade das superfícies articulares talonaviculares e naviculocuneiformes, assim como o comprimento e a estabilidade da coluna medial do pé e a inserção adequada do tendão tibial posterior. ${ }^{2,3,6,23}$ Embora algumas fraturas do escafoide possam ser tratadas de forma conservadora (aquelas sem deslocamento, com coluna medial de comprimento e estabilidade adequadas ao exame dinâmico e sem lesões associadas), as indicações para o tratamento cirúrgico são: incongruência articular $\geq 2 \mathrm{~mm}$; encurtamento da coluna medial $>3 \mathrm{~mm}$; incapacidade de obtenção ou manutenção da redução da articulação; fraturas expostas; presença de lesões múltiplas no médiopé; síndrome compartimental concomitante; e elevação da pele sobrejacente ao local da fratura ou em risco devido ao deslocamento da fratura. ${ }^{1-4}$ Além disso, o restauro de mais de $60 \%$ da superfície articular (visível nas radiografias anteroposterior e lateral) é fundamental para a estabilidade da articulação talonavicular. ${ }^{1,3}$ A RAFI cada vez mais se torna o padrão-ouro para fraturas articulares cominutivas com deslocamento e passíveis de reconstrução, como neste caso. ${ }^{3,4,24}$ Dependendo da cominuição da fratura, a realização de uma incisão mediana padrão ou dupla, que combina uma incisão mediana (entre os tendões dos músculos extensores digitais breve e longo ou lateral ao tendão do músculo extensor longo do hálux) e uma incisão anteromedial (especialmente nas fraturas do tipo III, como neste caso), é aconselhada e apoiada pela literatura recente. ${ }^{1-3,24}$ Na verdade, a abordagem dupla permite a melhor visualização e redução da fratura, e poupa os tecidos moles da retração excessiva, evitando complicações na cicatrização cutânea e óssea. ${ }^{3}$

O tratamento cirúrgico e a escolha do implante podem variar de acordo com o deslocamento, as lesões associadas, a estabilidade da coluna medial, a cominuição da superfície articular e a capacidade de reconstrução da fratura, bem como com os fatores comórbidos do paciente (por exemplo, diabetes, tabagismo intenso e comprometimento vascular) e a capacidade funcional geral. ${ }^{1-6,12,18}$ Não há recomendação formal para o momento ideal de realização da cirurgia; na ausência de indicações cirúrgicas urgentes, o procedimento deve ser feito com as condições apropriadas de diagnóstico por imagem e envelope de tecidos moles. ${ }^{3}$

Nas fraturas articulares com fragmentação e deslocamento, além de estabilidade da coluna medial, a RAFI com placas de travamento de minifragmentos é uma boa técnica de fixação, e teve bons resultados, com pontuação de 90,6 na escala AOFAS
20 semanas após a cirurgia. ${ }^{24,25} 0$ enxerto ósseo autólogo pode ser utilizado, pois seus efeitos osteocondutivos e osteoindutores facilitam a restauração anatômica e a cicatrização de fraturas..$^{2,4,26,27}$ No entanto, o enxerto ósseo não melhorou a convalescença nem o resultado clínico ou funcional; além disso, um estudo ${ }^{27}$ também relatou o tratamento de fraturas do calcâneo. Nas fraturas com instabilidade da coluna medial, como neste caso, pode-se considerar fixação externa adjunta à RAFI (com posicionamento no tálus e transfixação dos cuneiformes e do primeiro metatarso). Embora seu uso não seja suficientemente apoiado pela literatura atual (devido à baixa incidência dessas fraturas), sabe-se que, em alguns casos, ajuda a aumentar a estabilidade e manter a distração, permitindo a cicatrização da fratura. ${ }^{2-4,6}$ Nesse tipo de fratura, uma alternativa é a utilização de fixação de extensão interna, principalmente quando a compressão interfragmentar não é possível. A fixação de extensão interna tem os mesmos princípios de fixação, e pode atuar como contraforte ou neutralizar forças, ajudando a manutenção do comprimento e da estabilidade. Essa técnica é cada vez mais empregada devido ao desenvolvimento de novos materiais de fixação. ${ }^{2,3,6,28}$ Nas duas técnicas, a imobilização (com observação frequente das condições cutâneas, geralmente com uso de andador ou bota imobilizadora) e a restrição à sustentação de carga devem ocorrer por pelo menos seis semanas. ${ }^{1-6,28}$ Nesse período, o acompanhamento radiológico padronizado deve ser realizado para avaliação do alinhamento da coluna, do progresso da cicatrização, da falência do implante, da perda de redução, e do desenvolvimento de osteoartrite pós-traumática ou osteonecrose. $^{2-4}$ Após esse período, se nenhuma complicação for observada e o paciente não apresentar dor, o suporte parcial de peso deve ser permitido por quatro a seis semanas. A consolidação da fratura deve ocorrer de 10 a 12 semanas após a cirurgia. ${ }^{2}$ Se a consolidação não for vista com clareza nas radiografias comuns, uma TC deve ser solicitada, pois pode facilmente diagnosticar uma complicação da união da fratura. $^{3,25}$ Em comparação à ressonância magnética (lembrando que apenas pacientes tratados com implantes de titânio podem ser submetidos a essa técnica de imagem), a TC tem a vantagem de ser usada com qualquer tipo de implante. Além disso, os implantes de aço não são apenas seguros à TC: também estão associados a menos complicações se houver necessidade de remoção. ${ }^{3}$ Nos casos de complicações da união, um enxerto ósseo secundário e o prolongamento do tempo de imobilização podem ser considerados. ${ }^{2,3}$ Se houver sinais óbvios de consolidação, a fixação de extensão interna ou externa deve ser removida (geralmente de 10 a 12 semanas após a cirurgia, ou antes, se houver boa evolução clínica e radiológica), o que permite a restauração do movimento no complexo articular de Chopart e evita a rigidez; a seguir, a sustentação de peso deve ser permitida com cargas crescentes. $^{2,3,23-26}$

Em caso de acometimento das articulações naviculocuneiformes pela fratura, a fusão primária dessas articulações deve ser fortemente considerada, pois não são essenciais para o movimento do médiopé, e podem estabilizar a coluna medial; além disso, o procedimento não provoca incapacidade em longo prazo. ${ }^{1-4}$ Em pacientes com luxação complexa da 
fratura, geralmente uma fratura instável e deslocada sem possibilidade de reconstrução, a artrodese primária deve ser considerada. ${ }^{1-4}$ No entanto, a princípio, todo o possível deve ser feito para a reconstrução da superfície articular talonavicular, mesmo que não seja perfeita; a função do paciente deve ser avaliada e, se não for aceitável, a fusão talonavicular pode ser realizada. ${ }^{1}$

Apesar dos resultados satisfatórios e das altas taxas de união, as fraturas escafoides tendem a ter complicações e impacto funcional em longo prazo.

O tecido mole é um fator importante que pode influenciar o curso do tratamento e o resultado, como mostrado neste caso. Além da não união, abordada anteriormente, outras complicações, como osteoartrite pós-traumática, dor, rigidez, deformidade, infecção crônica e osteonecrose, podem provocar incapacidade em longo prazo. ${ }^{1-4} \mathrm{~A}$ dor não é incomum, e é uma das principais causas de comprometimento, sendo observada em quase um terço dos pacientes ${ }^{4}$; na maioria das vezes, é decorrente da ruptura ligamentar não diagnosticada, de lesões de cartilagem por impactação não reconhecidas, osteoartrite pós-traumática, impacto dorsal de calos excessivos, e intolerância ao implante., ${ }^{2,3}$ A osteoartrite pós-traumática é a sequela mais comum que complica uma fratura escafoide. De modo geral, é observada em fraturas complexas do corpo, principalmente naquelas que acometem a articulação talonavicular, e sua gravidade correlacionada é à congruência articular (se menos de $60 \%$ da articulação talonavicular for reconstruída). ${ }^{2,3}$ Por ser parte do complexo articular de Chopart e muito importante para a cinética do retropé e do médiopé, o movimento em uma superfície articular talonavicular danificada pode causar dor; como pode exigir uma fusão tardia para erradicar a dor, mas também provocar rigidez e perda de movimento do pé em logo prazo, isso precisa ser discutido com o paciente. $2,3,5,17,18,28$

Não união e osteonecrose podem ocorrer; ${ }^{3-6,19,24}$ esta última se deve especialmente à baixa vascularização da porção central do corpo do escafoide. ${ }^{10}$ Ao contrário da não união, a osteonecrose pode causar deformidade substancial, normalmente no alinhamento em varo do retropé, devido ao colapso do aspecto lateral do navicular em fraturas com deslocamento; isso é preocupante, e as queixas podem ser ainda mais graves do que a osteoartrite pós-traumática que a precede. Nesse caso, a correção do comprimento e do alinhamento deve ser considerada, geralmente por fusão talonavicular e/ou naviculocuneiforme, ou também por artrodese tripla, com interposição de enxerto ósseo, em casos graves. $^{1-4}$ Em um trabalho recente, ${ }^{29}$ o desenvolvimento de pé chato (21\%) e pé cavo (26\%) também foi relatado após fraturas de escafoide; deve ser dada atenção à ocorrência dessa deformidade que, se sintomática, deve ser tratada da maneira adequada.

O bom resultado alcançado neste caso pode ser atribuído à atenção dada aos aspectos particulares no manejo dessas lesões.

O caso também deve alertar para a crescente frequência dessas lesões, à medida que esportes radicais como o parkour ganham popularidade.
Conflitos de Interesse

Os autores declaram não haver conflitos de interesse.

\section{Referências}

1 Ramadorai MU, Beuchel MW, Sangeorzan BJ. Fractures and dislocations of the tarsal navicular. JAm Acad Orthop Surg 2016;24 (06):379-389

2 Schildhauer TA, Coulibaly MO, Hoffmann MF. Fractures and dislocations of the midfoot and forefoot. In: Court-Brown C, Heckman JD, McKee M, McQueen MM, Ricci W, Tornetta P 3rd, editors. Rockwood and Green's fractures in adults. 8th ed. Philadelphia: Wolters Kluwer; 2015:2689

3 DiGiovanni CW. Fractures of the navicular. Foot Ankle Clin 2004;9 (01):25-63

4 Sangeorzan BJ, Benirschke SK, Mosca V, Mayo KA, Hansen ST Jr. Displaced intra-articular fractures of the tarsal navicular. JBone Joint Surg Am 1989;71(10):1504-1510

5 Eichenholtz SN, Levine DB. Fractures of the tarsal navicular bone. Clin Orthop Relat Res 1964;34(34):142-157

6 Richter M, Wippermann B, Krettek C, Schratt HE, Hufner T, Therman H. Fractures and fracture dislocations of the midfoot: occurrence, causes and long-term results. Foot Ankle Int 2001;22 (05):392-398

7 Golano P, Fariñas O, Sáenz I. The anatomy of the navicular and periarticular structures. Foot Ankle Clin 2004;9(01):1-23

8 Astion DJ, Deland JT, Otis JC, Kenneally S. Motion of the hindfoot after simulated arthrodesis. J Bone Joint Surg Am 1997;79(02):241-246

9 McKeon KE, McCormick JJ, Johnson JE, Klein SE. Intraosseous and extraosseous arterial anatomy of the adult navicular. Foot Ankle Int 2012;33(10):857-861

10 Waugh W. The ossification and vascularisation of the tarsal navicular and their relation to Köhler's disease. JBone Joint Surg Br 1958;40-B(04):765-777

11 Court-Brown CM, Caesar B. Epidemiology of adult fractures: A review. Injury 2006;37(08):691-697

12 Pinney SJ, Sangeorzan BJ. Fractures of the tarsal bones. Orthop Clin North Am 2001;32(01):21-33

13 Wanke EM, Thiel N, Groneberg DA, Fischer A. [Parkour-“art of movement" and its injury risk]. Sportverletz Sportschaden 2013; 27(03):169-176

14 McLean CR, Houshian S, Pike J. Paediatric fractures sustained in Parkour (free running). Injury 2006;37(08):795-797

15 Miller JR, Demoiny SG. Parkour: a new extreme sport and a case study. J Foot Ankle Surg 2008;47(01):63-65

16 Coulibaly MO, Jones CB, Sietsema DL. Results of 90 consecutive navicular fractures. In: Paper presented at: AAOS 2010 Annual Meeting. 2010

17 Howie CR, Hooper G, Hughes SP. Occult midtarsal subluxation. Clin Orthop Relat Res 1986;(209):206-209

18 Meister K, Demos HA. Fracture dislocation of the tarsal navicular with medial column disruption of the foot. J Foot Ankle Surg 1994; 33(02):135-137

19 Rammelt S, Grass R, Zwipp H. [Nutcracker fractures of the navicular and cuboid]. Ther Umsch 2004;61(07):451-457

20 Myerson M. Foot and ankle disorders. Philadelphia, PA: Saunders; 2000

21 Rockett MS, Brage ME. Navicular body fractures: computerized tomography findings and mechanism of injury. JFoot Ankle Surg 1997;36(03):185-191

22 Haapamaki VV, Kiuru MJ, Koskinen SK. Ankle and foot injuries: analysis of MDCT findings. AJR Am J Roentgenol 2004;183(03): 615-622

23 Sangeorzan BJ, Hansen ST Jr. Early and late posttraumatic foot reconstruction. Clin Orthop Relat Res 1989;(243):86-91

24 Evans J, Beingessner DM, Agel J, Benirschke SK. Minifragment plate fixation of high-energy navicular body fractures. Foot Ankle Int 2011;32(05):S485-S492 
25 Cronier P, Frin JM, Steiger V, Bigorre N, Talha A. Internal fixation of complex fractures of the tarsal navicular with locking plates. A report of 10 cases. Orthop Traumatol Surg Res 2013;99(04):S241-S249

26 Schildhauer TA, Nork SE, Sangeorzan BJ. Temporary bridge plating of the medial column in severe midfoot injuries. JOrthop Trauma 2003;17(07):513-520

27 Longino D, Buckley RE. Bone graft in the operative treatment of displaced intraarticular calcaneal fractures: is it helpful? J Orthop Trauma 2001;15(04):280-286
28 Apostle KL, Younger AS. Technique tip: open reduction internal fixation of comminuted fractures of the navicular with bridge plating to the medial and middle cuneiforms. Foot Ankle Int 2008; 29(07):739-741

29 Coulibaly MO, Jones CB, Sietsema DL. Radiographic analysis of navicular fractures, [abstract number: 24250]. Gothenburg, Sweden: 7th SICOT/SIROT Annual International Conference; 2010 